\title{
Nanomedical strategy to prolong survival period, heighten cure rate, and lower systemic toxicity of SI 80 mice treated with MTX/MIT
}

This article was published in the following Dove Press journal:

Drug Design, Development and Therapy

30 August 2016

Number of times this article has been viewed

\author{
Ning Song' \\ Ming Zhao',2 \\ Yuji Wang' \\ $\mathrm{Xi} \mathrm{Hu}{ }^{\prime}$ \\ Jianhui $\mathrm{Wu}^{\prime}$ \\ Xueyun Jiang' \\ Shan $\mathrm{Li}^{1}$ \\ Chunying Cui' \\ Shiqi Peng'
}

'Beijing Area Major Laboratory of Peptide and Small Molecular Drugs, Engineering Research Center of Endogenous Prophylactic of Ministry of Education of China, Beijing Laboratory of Biomedical Materials, College of Pharmaceutical Sciences of Capital Medical University, Beijing, People's Republic of China; ${ }^{2}$ Department of Biomedical Science and Environmental Biology, Kaohsiung Medical University, Kaohsiung, Taiwan

Correspondence: Ming Zhao; Shiqi Peng Beijing Area Major Laboratory of Peptide and Small Molecular Drugs, Engineering Research Center of Endogenous Prophylactic of Ministry of Education of China, Beijing Laboratory of Biomedical Materials, College of Pharmaceutical Sciences of Capital Medical University, Beijing 100069 , People's Republic of China

Tel $+86108391 \quad 1528$

Fax +86 I0 839। I533

Email mingzhao@bjmu.edu.cn; sqpeng@bjmu.edu.cn

\begin{abstract}
In spite of the usual combination form of methotrexate (MTX)/mitoxantrone (MIT) and various complex combination regimens of MTX/MIT with other anticancer drugs, the survival period, cure rate, and systemic toxicity still need to be improved. For this purpose, a nanostructured amino group-modified mesoporous silica nanoparticles (MSNN)-MTX/MIT was designed. In the preparation, the surface of mesoporous silica nanoparticles (MSNs) was modified with amino groups to form MSNN. The covalent modification of the amino groups on the surface of MSNN with MTX resulted in MSNN-MTX. The loading of MIT into the surface pores of MSNN-MTX produced nanostructured MSNN-MTX/MIT. Compared with the usual combination form (MTX/MIT), nanostructured MSNN-MTX/MIT increased the survival period greatly, heightened the cure rate to a great extent, and lowered the systemic toxicity of the treated S180 mice, significantly. These superior in vivo properties of nanostructured MSNN-MTX/MIT over the usual combination form (MTX/MIT) were correlated with the former selectively releasing MTX and MIT in tumor tissue and inside cancer cells in vitro. The chemical structure and the nanostructure of MSNN-MTX/MIT were characterized using infrared and differential scanning calorimeter spectra as well as transmission electron microscope images, respectively.
\end{abstract}

Keywords: mitoxantrone, methotrexate, mesoporous silica nanoparticles, cancer therapy, nanomedicine

\section{Introduction}

Methotrexate (MTX) and mitoxantrone (MIT) exhibit antitumor actions in vitro and in vivo, ${ }^{1-4}$ as well as possess in vivo activity, which aids in treating multiple sclerosis, ${ }^{5}$ but serious side effects of these drugs greatly limit the therapeutic efficacy. The side effects of MTX are stomatitis and alimentary canal bleeding, ${ }^{6,7}$ while MIT causes hematological and hepatic adverse effects. ${ }^{8,9}$ To prolong the survival period, heighten the cure rate, and reduce the systemic toxicity of MTX/MIT in treating cancer patients, various combination regimens, such as MIT/MTX/mitomycin followed by cisplatin/ gemcitabine for treating unresectable malignant pleural mesothelioma, ${ }^{10} \mathrm{MTX} /$ MIT/mitomycin C for treating advanced breast cancer, ${ }^{11-14} \mathrm{MIT} /$ cyclophosphamide/ MTX/5-flurouracil/prednisone for treating advanced breast cancer, ${ }^{15} \mathrm{MIT} / \mathrm{MTX} /$ folinic $\mathrm{acid} /$ methylprednisolone/vincristine/granulocyte for treating acute lymphoblastic leukemia, ${ }^{16}$ carmustine/doxorubicin/etoposide/vincristine/cyclophosphamide/MIT/ cytarabine/MTX/citrovorum factor for treating non-Hodgkin lymphoma, ${ }^{17}$ and MIT/ MTX/citrovorum for treating either metastatic or locally advanced transitional cell carcinoma of the bladder, ${ }^{18}$ have been used. Besides, MTX- and MIT-related combinations 


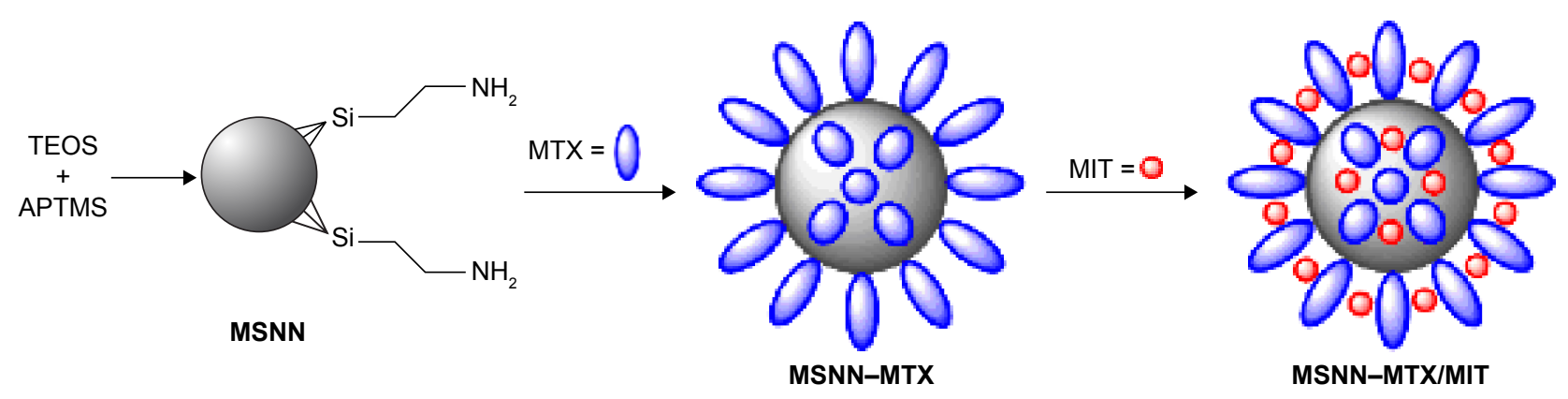

Figure I Preparation of MSNN-MTX/MIT.

Notes: The blue particle represents $\mathrm{NH}_{2}$ groups. The red particle represents MIT.

Abbreviations: MSN, mesoporous silica nanoparticles; MSNN, $\mathrm{NH}_{2}$-modified MSN; MTX, methotrexate; MIT, mitoxantrone; TEOS, tetraethyl orthosilicate; APTMS, 3-trimethoxysilyl-propane-I-amine on MSNN.

were used to reduce the toxicity of other anticancer drugs, such as MTX/MIT/mitomycin C combination being used to reduce nausea, vomiting, alopecia, and cardiotoxicity of doxorubicin-based protocols. ${ }^{19}$ Similar to the usual combination form (MTX/MIT), however, the survival period, the cure rate, and the systemic toxicity of the aforementioned combination regimens ${ }^{20,21}$ still need to be improved.

It is recognized commonly that loading a drug onto delivery vehicle leads to a decrease in toxicity. ${ }^{22-25}$ Among the various nanomaterials, mesoporous silica nanoparticles (MSNs) have an ordered structure, numerous holes, desirable hole size, acceptable biocompatibility, and biodegradability. ${ }^{26}$ Functional modification of the surface of MSNs results in the establishment of a nanomedical platform either for various needs (such as imaging, diagnostics, and clinical therapy) ${ }^{27}$ or for abolishing the side effects of the anticancer drugs in particular. ${ }^{28}$ In this context, we modified the surface of MSNs with amino groups to prepare amino groups-modified mesoporous silica nanoparticles (MSNN), covalently linked MTX with the amino groups of MSNN to prepare MSNN-MTX, and loaded MIT into the surface pores to prepare MSNNMTX/MIT (Figure 1). Thus we used the nanomedical strategy of combining MTX and MIT rather than a usual combination form (MTX/MIT), for treating S180 mice, thereby showing the superiority of MSNN-MTX/MIT over MTX/MIT in the survival period, the cure rate, and the systemic toxicity.

\section{Experimental section}

\section{Preparing MSNN and MSNN-MTX}

Based on previous literature, MSNN was prepared. ${ }^{7}$ For the preparation of MSNN-MTX (MTX covalently modified MSNN), a solution of $285 \mathrm{mg}(1.49 \mathrm{mmol})$ of $N$-ethyl- $N^{\prime}$-(3dimethyl-aminopropyl)-carbodiimide hydrochloride in $10 \mathrm{~mL}$ of anhydrous dimethyl sulfoxide was mixed with $95.7 \mathrm{mg}$ (0.21 mmol) of MTX. Into this mixture, $275 \mathrm{mg}$ of MSNN was added and the suspension stirred at room temperature for 24 hours to form MSNN-MTX. After centrifugation, the precipitates were successively washed with dimethyl sulfoxide, water, and ethanol to completely remove free MTX, ie, no MTX should be detected in the supernatant by absorption spectroscopy (Shimadzu UV-2550 spectrophotometer, Kyoto, Japan, $306 \mathrm{~nm}$ ), and dried in vacuum for 24 hours. The content of covalently conjugated MTX was also identified using absorption spectroscopy.

\section{Preparing MSNN-MTX/MIT}

To a solution of $100 \mathrm{mg}$ of MIT in $2 \mathrm{~mL}$ of ultrapure water, $1 \mathrm{~g}$ of MSNN-MTX was added. The suspension was ultrasonically oscillated for 20 minutes at room temperature and centrifuged at $120 \mathrm{rpm}$ for 15 minutes; then, the collected precipitates were dried for loading. This loading procedure was repeated for five times by ultrasonically oscillating the dried precipitates in a solution of $100 \mathrm{mg}$ MIT in $2 \mathrm{~mL}$ of ultrapure water to provide the MSNN-MTX/MIT. Gravimetric analysis showed that $1 \mathrm{~g}$ of MSNN-MTX/MIT contained $20 \mathrm{mg}$ of MIT.

\section{Measuring the release of MTX from MSNN-MTX}

The release of MTX from $6.0 \mathrm{mg}$ of MSNN-MTX was tested in $50 \mathrm{~mL}$ of phosphate-buffered solution (PBS; pH: 5.5, $\left.37^{\circ} \mathrm{C}\right)$ with $\alpha$-chymotrypsin $(0.1 \mathrm{mg} / \mathrm{mL})$. To alleviate the limitation of the release rate by external diffusion constraints, a constant rotation speed $(120 \mathrm{rpm} / \mathrm{min}$ ) was maintained during the test. The PBS was sampled and measured at $0.5,1,2$, $3,4,6,8,10,12,24,36,51,60,72,84$, and 96 hours.

\section{Measuring the release of MIT from MSNN-MTX/MIT}

The release of MIT from $20.0 \mathrm{mg}$ of MSNN-MTX/MIT was tested in $50 \mathrm{~mL}$ PBS $\left(37^{\circ} \mathrm{C}, \mathrm{pH}: 5.5\right.$ and 7.45$)$. To alleviate the limitation of the release rate by external diffusion constraints, a constant rotation speed $(120 \mathrm{rpm} / \mathrm{min})$ was maintained during 
the test. The PBS was sampled and measured at 1, 3, 6, 9, 12 , $24,36,48,60,72,96,120,144,180,204$, and 240 hours.

\section{Measuring TEM images of MSNN-MTX/MIT}

The nanostructures of MSN, MSNN-MTX, and MSNNMTX/MIT were visualized on a transmission electron microscope (TEM; JSM-6360 LV, JEOL). Approximately $1 \mathrm{mg}$ solution of MSNN or MSNN-MTX or MSNN-MTX/MIT in $1.5 \mathrm{~mL}$ of alcohol was added dropwise onto a formvarcoated copper grid, and then a drop of anhydrous ethanol was added to enhance the removal of water. Then, the grid was allowed to thoroughly dry in air and was then incubated at $37^{\circ} \mathrm{C}$ for 24 hours. The copper grids were viewed under a TEM and the nanostructures determined by counting over 100 species in randomly selected regions on the copper grids. All determinations were carried out in triplicate. The TEM was operated with an $80 \mathrm{kV}$ electron beam. Images were recorded on an imaging plate (Gatan Bioscan camera model 1792, Gatan, Pleasanton, CA, USA) with a $20 \mathrm{eV}$ energy window at $6,000-400,000 \times$ and were digitally enlarged.

\section{Measuring the DSC spectra of MSNN-MTX/MIT}

DSC (differential scanning calorimeter) spectra of MSNN, MIT, MTX, MSNN+MTX, MSNN-MTX, MSNN-MIT, and MSNN-MTX/MIT were recorded on a DSC (NETZSCH, 204F1). Approximately $1 \mathrm{mg}$ sample of MSNN or MIT or MTX or MSNN+MTX or MSNN-MTX or MSNN-MIT or MSNN-MTX/MIT was added into aluminum crucible and analyzed at $20^{\circ} \mathrm{C}-300^{\circ} \mathrm{C}$.

\section{Measuring the IR spectra of MSNN-MTX/MIT}

The infrared (IR) spectra of MSNN, MIT, MTX, MSNN+MTX, MSNN-MTX, MSNN-MIT, and MSNNMTX/MIT were recorded using a FT-IR spectrometer (Thermo Nicolet IS5, Thermo Fisher Scientific, Waltham, MA, USA). Approximately $1 \mathrm{mg}$ sample of MSNN or MIT or MTX or MSNN+MTX or MSNN-MTX or MSNN-MIT or MSNN-MTX/MIT was measured using the $\mathrm{KBr}$ pellet method at room temperature.

\section{Particle size and zeta potential of MSNN-MTX/MIT}

The particle size and zeta potential $(\zeta)$ potential of water (blank), MSNN, MSNN-MTX, and MSNN-MTX/MIT were measured using an instrument, Zeta PlusPotential Analyzer from Brookhaven Instruments Corporation (Holtsville, NY, USA) at room temperature. All samples were prepared by dissolving $1 \mathrm{mg}$ of sample in $1.5 \mathrm{~mL}$ of water, and the experiments were repeated individually for three times.

\section{In vivo tumor growth assays}

Male Institute of Cancer Research (ICR) mice (6 weeks old, 18-22 g) were purchased from the Animal Center of Capital Medical University and housed in an air-conditioned room.

The care and handling of the animals were performed with the approval of Institutional Authority for Laboratory Animal Care of Capital Medical University and adhered to these guidelines. S180 cells were purchased from Vital River Laboratory Animal Technology Co., Ltd. Beijing, People's Republic of China and xenografted subcutaneously in the mice. The mice were housed in sterile isolated cages at constant temperatures $\left(22^{\circ} \mathrm{C}-25^{\circ} \mathrm{C}\right)$ and given free access to food and water. Tumor growth was monitored by measuring the perpendicular diameter of the tumors using a caliper. Tumor volume was calculated using the formula, Volume $=$ Length $\times$ Width $2 / 2 \mathrm{~cm}^{3}$. When the average volume of tumors xenograft reached $0.5 \mathrm{~cm}^{3}$, the mice were randomly divided into sodium carboxyl methyl cellulose (CMCNa; 0.5\%, $10 \mathrm{~mL} / \mathrm{kg} / \mathrm{d}$ ) group, MTX (6 mg/kg/d) + MIT (1 mg/kg/d) group, and MSNN-MTX/MIT (50 mg/kg/d) group, each with 12 mice. The mice were then intraperitoneally injected with $\mathrm{CMCNa}$, MTX+MIT, and MSNN-MTX/MIT for 7 consecutive days. The mice were bred for another 6 days and then sacrificed for further investigations, such as survival analysis, tumor volume determination, and tumor weight test.

\section{Liver/kidney toxicity assays Plasma ALT and AST assays}

Approximately $0.9 \mathrm{~mL}$ of blood of the mice receiving antitumor assay was collected into a syringe containing $0.1 \mathrm{~mL}$ of $3.8 \%$ sodium citrate and immediately centrifuged at 3,000 rpm for 10 minutes to get the plasma samples. The separated plasma was used to measure plasma levels of alanine transaminase (ALT) and aspartate transaminase (AST) according to the manufacturer's instructions (AST/GOT testing kit, ALT/GPT testing kit; JCBIO Co., Nanjing, People's Republic of China). In brief, $5 \mathrm{~mL}$ of plasma was added into the test wells of 96 microtiter plates, then $20 \mathrm{~mL}$ of Matrix liquid was added and incubated at $37^{\circ} \mathrm{C}$ for 30 minutes; then, $5 \mathrm{~mL}$ of plasma was added into the control wells of the 96 microtiter plates followed by addition of $20 \mathrm{~mL}$ of 2,4-dinitrophenylhydrazine to all wells. The plates were incubated at $37^{\circ} \mathrm{C}$ for 20 minutes. Then, $200 \mathrm{~mL}$ 
of $0.4 \mathrm{~mol} \mathrm{~L}{ }^{-1} \mathrm{NaOH}$ was added and incubated at $37^{\circ} \mathrm{C}$ for 15 minutes, and the absorbance of the yellow solutions was recorded at $510 \mathrm{~nm}$ to calculate vitality using the formula: $\left(\mathrm{OD}_{\text {test }}-\mathrm{OD}_{\text {control }}\right) / K_{\text {curve }}$, where $\mathrm{OD}_{\text {test }}$ refers to the absorbance of the test well, $\mathrm{OD}_{\text {control }}$ refers to the absorbance of the control well, and $K_{\text {curve }}$ refers to the standard curve, which is calculated using the absorbance of the standard well following the manufacturer's protocols.

\section{Plasma Cr assay}

Approximately $0.9 \mathrm{~mL}$ of blood of the mice receiving antitumor assay was collected into a syringe containing $0.1 \mathrm{~mL}$ of $3.8 \%$ sodium citrate and immediately centrifuged at 3,000 rpm for 10 minutes to get the plasma samples. The separated plasma was used to measure plasma levels of creatinine $(\mathrm{Cr})$ according to the guidelines provided in the kit (Cr testing kit; JCBIO Co., Nanjing, People's Republic of China). In brief, $6 \mathrm{~mL}$ of plasma or standard solution was added into the wells of 96 microtiter plates; then, $180 \mathrm{~mL}$ of the enzyme solution A was added and incubated at $37^{\circ} \mathrm{C}$ for 5 minutes followed by addition of $60 \mathrm{~mL}$ of the enzyme solution B. The samples were again incubated at $37^{\circ} \mathrm{C}$ for 5 minutes, and the absorbance of the purple solutions was recorded at $546 \mathrm{~nm}$ to calculate vitality using the formula: $\left(\mathrm{OD}_{\text {test }}-\mathrm{OD}_{\text {control }}\right) / K_{\text {curve }}$, where $\mathrm{OD}_{\text {test }}$ refers to the absorbance of the test well, $\mathrm{OD}_{\text {control }}$ refers to the absorbance of the control well, and $K_{\text {curve }}$ refers to the standard curve.

\section{Results and Discussion}

As depicted in Figure 1, the reaction of 3-trimethoxysilylpropane-1-amine resulted in the formation of MSNN. The reaction between MSNN and MTX formed MSNN-MTX, ie, MTX covalently modified MSNN. UV analysis showed that $1 \mathrm{~g}$ of MSNN-MTX contained $120 \mathrm{mg}$ of MTX. By repeated absorptions, more MIT was loaded into the holes of MSNN-MTX to form MSNN-MTX/MIT (20 mg MIT/g MSNN-MTX/MIT). Gravimetric analysis showed that $1 \mathrm{~g}$ of MSNN-MTX/MIT contained $20 \mathrm{mg}$ of MIT.

\section{In vivo activities of MSNN-MTX/MIT and MTX/MIT}

In vivo antitumor evaluations were performed on male ICR mice. In brief, S180 cells were xenografted subcutaneously in mice housed in sterile isolated cages at $22^{\circ} \mathrm{C}-25^{\circ} \mathrm{C}$. Tumor growth was monitored by measuring the perpendicular diameter of the tumors using a caliper. Tumor volume was calculated using the formula, Volume $=$ Length $\times \mathrm{Width}^{2} / 2 \mathrm{~cm}^{3}$. Seven days after the xenograft of S180 cells, the volume of the tumor reached $\sim 0.5 \mathrm{~cm}^{3}$ and the mice were randomly divided into three groups of 12 mice each. The mice were intraperitoneally injected with $\mathrm{CMCNa}(0.5 \%, 10 \mathrm{~mL} / \mathrm{kg} / \mathrm{d})$, MTX (6 mg/kg/d)/MIT (1 mg/kg/d), and MSNN-MTX/ MIT $(50 \mathrm{mg} / \mathrm{kg} / \mathrm{d})$ for 7 consecutive days. The mice were bred for an additional 6 days and then sacrificed for further investigations.

\section{Systemic toxicity and survival period of SI 80 mice treated with MSNN-MTXI MIT and MTX/MIT}

Lethality and the ratio of organ/bodyweight are used to indicate systemic toxicity and survival period after therapy. Figure 2 shows the lethality during a 13-day period in which S180 mice received CMCNa, MSNN-MTX/MIT, and MTX/ MIT at the same dose. The lethality of CMCNa- or MSNNMTX/MIT-treated S180 mice on day 13 was $0 \%$, while the lethality of MTX/MIT-treated S180 mice on days 6, 7, and 8 was $17 \%, 67 \%$, and $100 \%$, respectively. The significant lethality suggests that for prolonging survival period, nanostructured MSNN-MTX/MIT is superior over the usual combination form (MTX/MIT).

Table 1 shows the body weight and the ratios of organ weight/body weight of S180 mice receiving nanostructured MSNN-MTX/MIT and the usual combination form (MTX/ MIT) at the same dose. The body weight and the ratios of liver, spleen, brain, and kidney weights to body weight of the mice that received MTX/MIT were significantly different from those of the mice that received CMCNa or MSNNMTX/MIT, while the body weight and the ratios of liver,

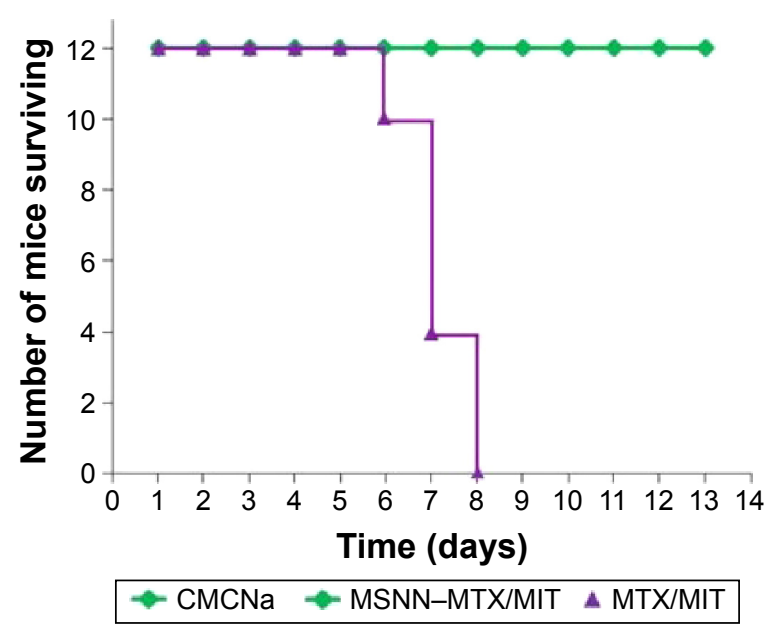

Figure 2 Survival of SI 80 mice treated with CMCNa, MTX/MIT, and MSNN-MTX/ MIT during the 7-day administration and the 13-day observation period. Abbreviations: $\mathrm{CMCNa}$, sodium carboxyl methyl cellulose; MTX, methotrexate; MIT, mitoxantrone; MSNN, amino group-modified mesoporous silica nanoparticles. 
Table I Body weight and ratios of organ weight/body weight of MSNN-MTX/MIT- and MTX/MIT-treated SI 80 mice

\begin{tabular}{llllll}
\hline Group & Body weight $(\mathrm{g})$ & Liver/body $(\%)$ & Spleen/body (\%) & Brain/body (\%) & Kidney/body (\%) \\
\hline CMCNa & $41.85 \pm 1.79$ & $6.95 \pm 0.59$ & $1.00 \pm 0.26$ & $0.74 \pm 0.16$ & $0.49 \pm 0.08$ \\
MTX/MIT & $21.23 \pm 1.80^{\mathrm{a}}$ & $4.72 \pm 0.76^{\mathrm{a}}$ & $0.22 \pm 0.07^{\mathrm{a}}$ & $1.09 \pm 0.57^{\mathrm{a}}$ & $0.70 \pm 0.10^{\mathrm{a}}$ \\
MSNN-MTX/MIT & $37.85 \pm 2.20$ & $7.33 \pm 0.61$ & $0.93 \pm 0.22$ & $0.71 \pm 0.08$ & $0.47 \pm 0.13$ \\
\hline
\end{tabular}

Notes: All values are mean \pm SD. a Compared to CMCNa group, $P<0.01$; two, six, and four mice $(n=12)$ in MTX/MIT group died on days 6 , 7, and 8 , respectively. The bodies and organs were weighed immediately after the death of the animals.

Abbreviations: MTX, methotrexate; MIT, mitoxantrone; MSNN, amino group-modified mesoporous silica nanoparticles; CMCNa, sodium carboxyl methyl cellulose; $\mathrm{SD}$, standard deviation.

spleen, brain, and kidney weights to body weight of the mice that received CMCNa were at the same level as those of the mice that received MSNN-MTX/MIT. This suggests that treatment for even 6,7 , and 8 days with the usual combination form (MTX/MIT) still significantly slowed the growth and damaged the liver, spleen, brain, and kidney of the mice. In contrast, 13-day treatment with the nanostructured MSNNMTX/MIT did not slow the growth and damage the liver, spleen, brain, and kidney of the mice. Therefore, for limiting systemic toxicity, the nanostructured MSNN-MTX/MIT was superior over the usual combination form (MTX/MIT).

\section{Tumor volume of SI80 mice treated with MSNN-MTX/MIT and MTX/MIT}

The superiority of nanostructured MSNN-MTX/MIT over the usual combination form (MTX/MIT) was demonstrated by comparing the tumor volume of S180 mice treated with the two different regimens. The treatment with MTX/MIT for 8 days induced all mice to die, and so the tumor volume of the mice treated for 13 days was unavailable (Figure 3). On day 13 , however, the tumor volume of CMCNa-treated

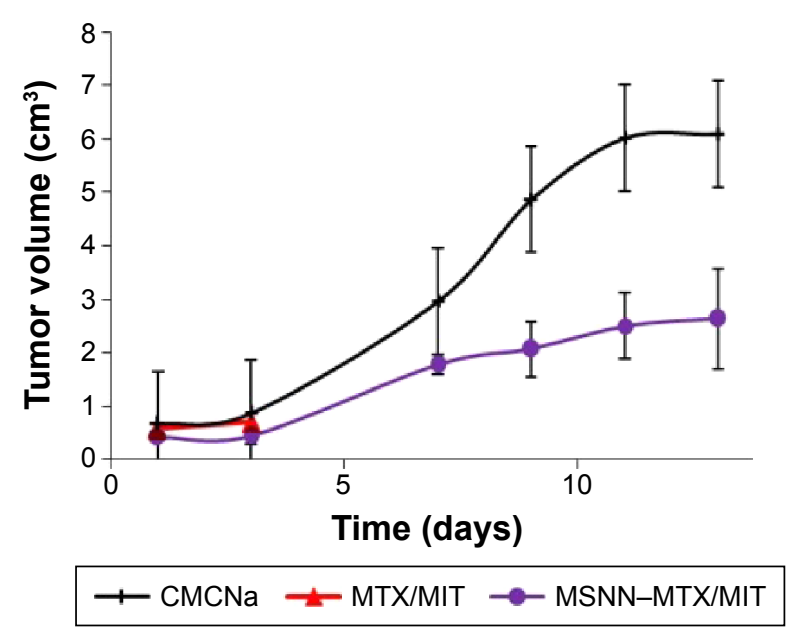

Figure 3 Tumor volume of SI80 mice treated with CMCNa, MTX/MIT, and MSNN-MTX/MIT

Note: $n=12$.

Abbreviations: $\mathrm{CMCNa}$, sodium carboxyl methyl cellulose; MTX, methotrexate; MIT, mitoxantrone; MSNN, amino group-modified mesoporous silica nanoparticles. mice was greater than twofold that of the mice treated with MSNN-MTX/MIT. Therefore, at the same dose as the usual combination form (MTX/MIT), nanostructured MSNNMTX/MIT effectively slowed tumor growth and was found to be superior over the usual combination form (MTX/MIT) in tumor therapy.

\section{Tumor weight of $\mathrm{S} 180$ mice treated with MSNN-MTX/MIT and MTX/MIT}

The superiority of nanostructured MSNN-MTX/MIT over the usual combination form (MTX/MIT) was mirrored with the tumor weight of the treated S180 mice. The treatment of MTX/MIT for 8 days induced all mice to die, and so the tumor weight of the mice treated for 13 days was unavailable. On day 13, the tumor weight of the mice treated with CMCNa was significantly higher than that of the mice treated with MSNN-MTX/MIT at the same dose as the usual combination form (MTX/MIT; Figure 4). Again, at the given dose, nanostructured MSNN-MTX/MIT effectively slowed tumor growth and was found to be superior over the usual combination form (MTX/MIT) in tumor therapy.

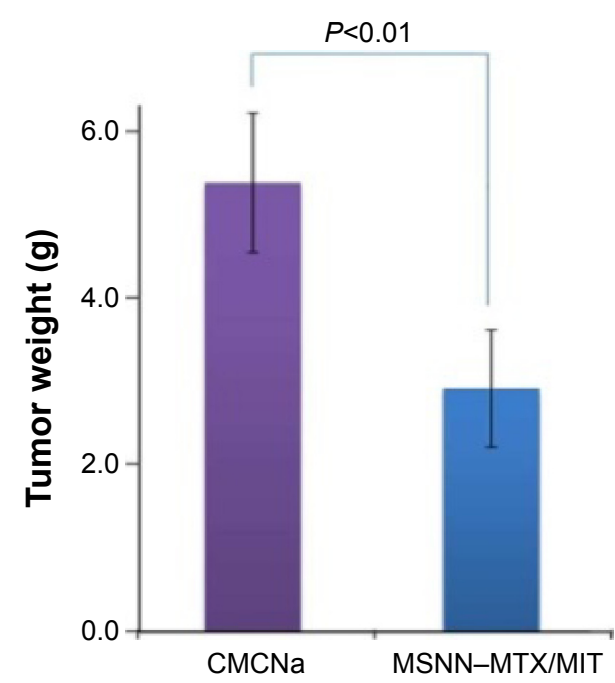

Figure 4 Tumor weights of CMCNa- and MSNN-MTX/MIT-treated mice. Note: $\mathrm{n}=12$.

Abbreviations: CMCNa, sodium carboxyl methyl cellulose; MTX, methotrexate; MIT, mitoxantrone; MSNN, amino group-modified mesoporous silica nanoparticles. 


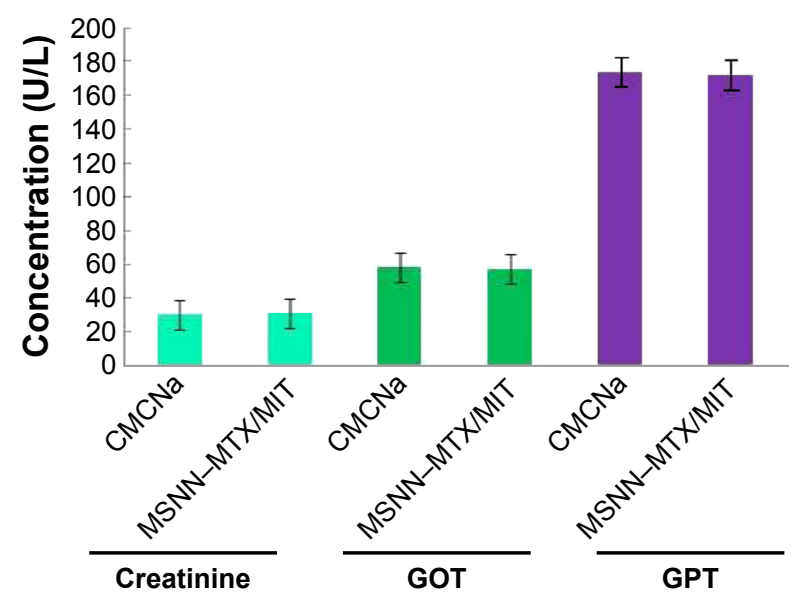

Figure 5 Serum ALT, AST, and Cr levels of the mice treated with CMCNa, MTX/ MIT, and MSNN-MTX/MIT.

Abbreviations: ALT, alanine transaminase; AST, aspartate transaminase; $\mathrm{Cr}$, creatinine; CMCNa, sodium carboxyl methyl cellulose; MTX, methotrexate; MIT, mitoxantrone; MSNN, amino group-modified mesoporous silica nanoparticles; GOT, glutamic oxaloacetic transaminase; GPT, glutamic pyruvic transaminase.

\section{Liver and kidney toxicity of SI 80 mice treated with MSNN-MTX/MIT and MTX/MIT}

The superiority of nanostructured MSNN-MTX/MIT over the usual combination form (MTX/MIT) was also mirrored by the serum ALT, AST, and Cr levels of the treated S180 mice. Treatment with the usual combination form (MTX/MIT) for 8 days induced all S180 mice to die, and so the levels of serum ALT, AST, and Cr on day 13 were unavailable. The levels of serum ALT, AST, and Cr on day 13 in the S180 mice treated with CMCNa were equal to those of the S180 mice treated with MSNN-MTX/MIT at the same dose as the usual combination form (MTX/MIT)
(Figure 5). The serum levels of ALT and AST indicate liver toxicity, and the serum level of $\mathrm{Cr}$ represents kidney toxicity. The fact that $\mathrm{S} 180$ mice treated with $\mathrm{CMCNa}$ and MSNN-MTX/MIT, showed equal serum levels of ALT, AST, and Cr, implies that nanostructured MSNN-MTX/ MIT caused no liver and kidney toxicity and is superior to the usual combination form (MTX/MIT) in limiting liver and kidney toxicity.

\section{Release property of MSNN-MTX and MSNN-MTX/MIT}

To understand the advantage of nanostructured MSNNMTX/MIT in prolonging the survival period, heightening the cure rate, and limiting the toxicity of the treated S180 mice, the in vitro release of MSNN-MTX and MSNN-MTX/ MIT was examined.

\section{MSNN-MTX and MSNN-MTX/MIT releasing $M T X$}

The release of MTX from 6 mg of MSNN-MTX was carried out in $50.0 \mathrm{~mL}$ of $\mathrm{PBS}\left(37^{\circ} \mathrm{C}, \mathrm{pH}: 7.45\right.$ and 5.5) with or without protease. The cumulative release was monitored by sampling the PBS at $0.5,1,2,3,4,6,8,10,12,24,36$, $51,60,72,84$, and 96 hours, and the curves are shown in Figure 6. In pH 7.45 and 5.5 PBS, MSNN-MTX released less than $10 \%$ of MTX at all time points; however, in $\mathrm{pH} 5.5$ PBS with $0.5 \mathrm{mM}$ of $\alpha$-chymotrypsin, the release of MTX was rapidly increased at 35 hours and reached the maximum at 70 hours, exhibiting a prolonged release manner.

The release of MTX from $15.0 \mathrm{mg}$ of MSNN-MTX/MIT was carried out in $50.0 \mathrm{~mL}$ PBS $\left(37^{\circ} \mathrm{C}\right.$, pH: 7.45 and 5.5) with
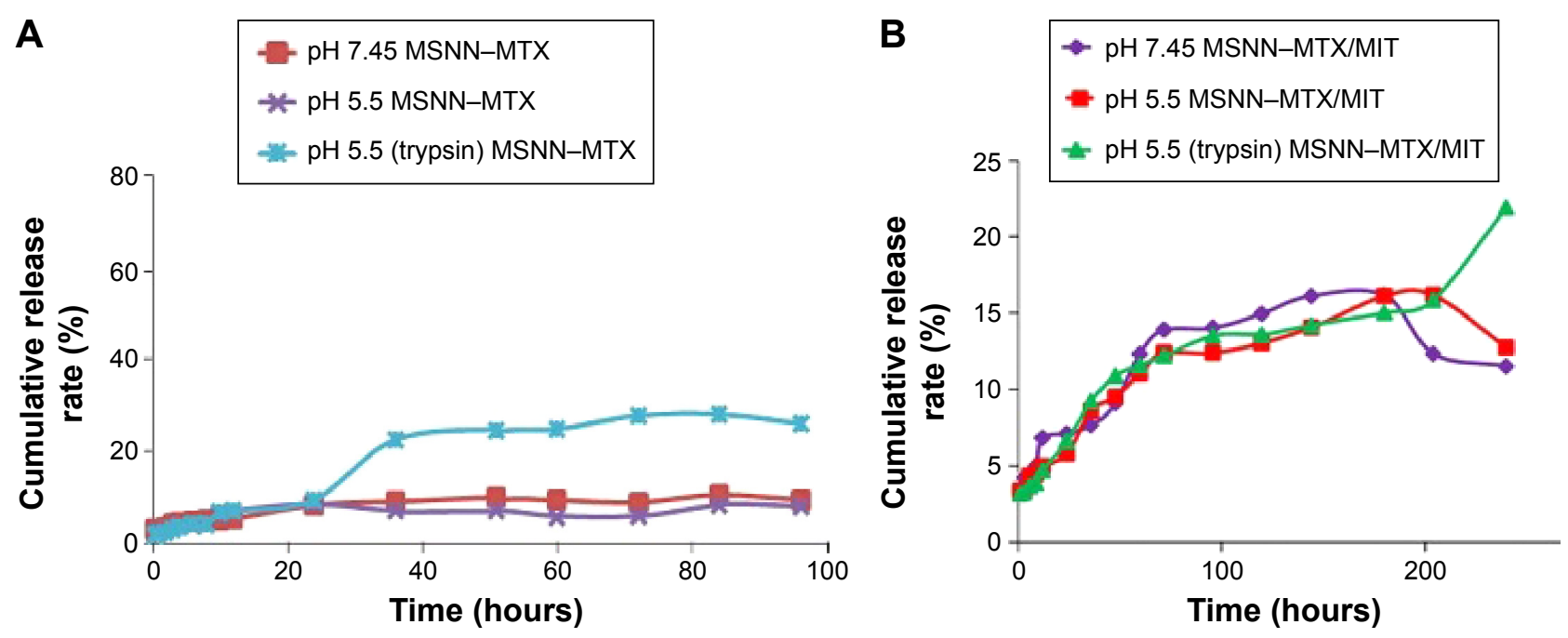

Figure $6 \alpha$-Chymotrypsin induces the cumulative release profile of MTX from (A) MSNN-MTX and (B) MSNN-MTX/MIT in pH 5.4 PBS.

Abbreviations: MTX, methotrexate; MIT, mitoxantrone; MSNN, amino group-modified mesoporous silica nanoparticles; PBS, phosphate-buffered solution. 


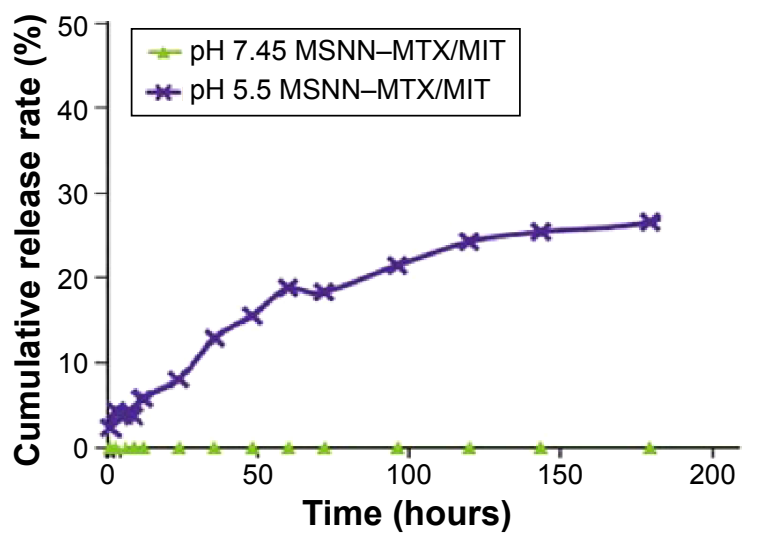

Figure 7 MSNN-MTX/MIT releases MIT in a time- and $\mathrm{pH}$-dependent manner. Abbreviations: MTX, methotrexate; MIT, mitoxantrone; MSNN, amino groupmodified mesoporous silica nanoparticles.

or without $\alpha$-chymotrypsin $(0.1 \mathrm{mg} / \mathrm{mL})$, and these curves are shown in Figure 6. Within 200 hours, in pH 5.5 and 7.45 PBS, MSNN-MTX/MIT slowly released MTX, and at 200 hours the release rate rapidly decreased. Although within 200 hours in pH 5.5 PBS with $0.5 \mathrm{mM}$ of $\alpha$-chymotrypsin, MSNN-MTX/ MIT slowly released MTX, at 200 hours the release rate of MTX rapidly increased, and this was in a controlled manner.

It is well documented that the tumor tissue $\mathrm{pH}$ value is 5.5 and the level of $\alpha$-chymotrypsin inside cancer cells is higher than that of $\alpha$-chymotrypsin outside cancer cells. ${ }^{29,30}$ Therefore, the observations suggest that inside tumor tissue, but not inside normal tissue, and inside cytosol of cancer cells, but not outside cytosol of cancer cells, MTX will be optionally released from MSNN-MTX and MSNNMTX/MIT. The loading of MIT onto the surface holes of MSNN-MTX changes the release manner, but does not influence the release of MTX.

\section{MSNN-MTX/MIT releasing MIT in $\mathrm{pH}$} 5.5 and 7.45 PBS

The release of MIT from $15.0 \mathrm{mg}$ of MSNN-MTX/MIT was carried out in $50.0 \mathrm{~mL}$ PBS (pH: 7.45 and 5.5 ) at $37^{\circ} \mathrm{C}$ by sampling the PBS at 1, 3, 6, 9, 12, 24, 36, 48, 60, 72, 96, 120, 144, and 180 hours, and the curves are shown in Figure 7. In $\mathrm{pH}$ 7.45 PBS, no MIT was released, suggesting that in the $\mathrm{pH}$ environment of blood and healthy tissues, MSNN-MTX/ MIT does not release MIT. ${ }^{7}$ In pH 5.5 PBS, however, with prolonged duration, MIT is gradually released, suggesting that in the $\mathrm{pH}$ environment of tumor tissues MSNN-MTX/ MIT releases MIT in a time-dependent manner. ${ }^{31}$

Nanostructured MSNN-MTX/MIT selectively releasing both MTX and MIT in tumor tissue, but not in normal organ tissue, would be responsible for prolonged survival period, heightened cure rate, and lowered systemic toxicity. Therefore, the release profiles of MSNN-MTX/MIT in tumor evidence the advantage of the nanostructured regimen.

\section{Nanoproperty of MSNN-MTX/MIT}

The size, shape, and surface feature of the nanoparticles of MSNN-MTX/MIT were studied. The images were recorded on a TEM (JEOL-200CX, Tokyo, Japan) and are shown in Figure 8. The TEM images indicate that the diameter of the nanoparticles of MSN ranged from 96 to $112 \mathrm{~nm}$ and the diameter of the surface holes was $\sim 2.5 \mathrm{~nm}$ (Figure 8A). The TEM images indicate that the diameter of the nanoparticles of MSNN-MTX was 94-116 nm and the diameter of the surface holes was $\sim 2.0 \mathrm{~nm}$ (Figure $8 \mathrm{~B}$ ). The TEM images indicate that the diameter of the nanoparticles of MSNN-MTX/MIT was 75-101 nm and the diameter of
A

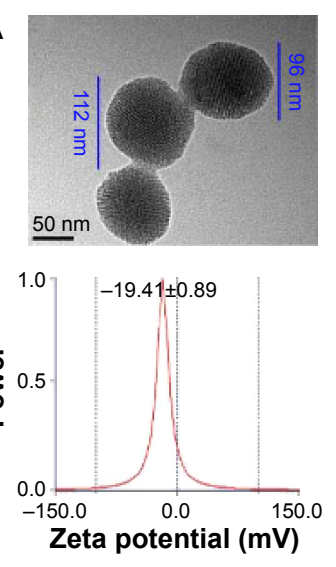

B
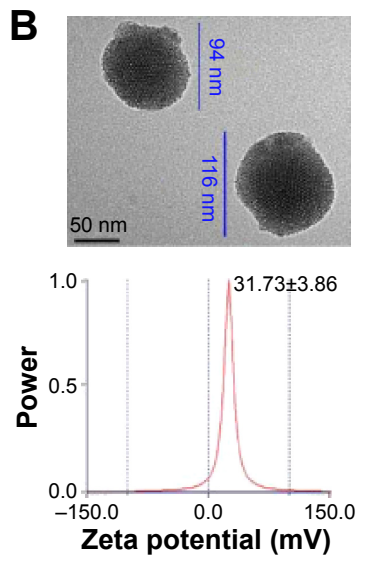

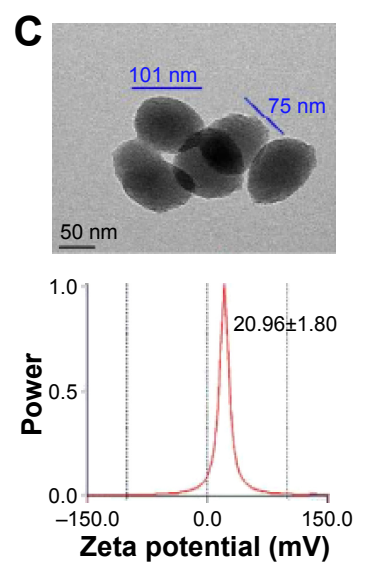

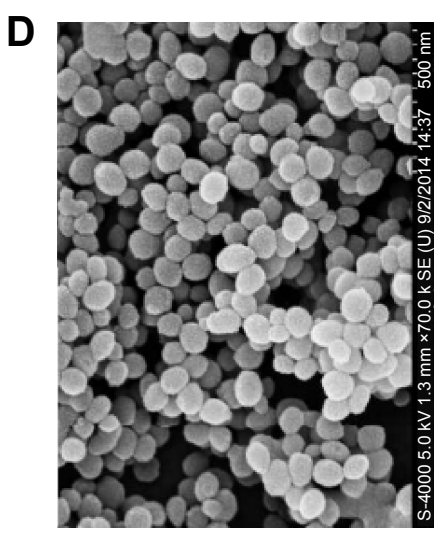

Figure 8 TEM images and $\zeta$ potentials.

Notes: (A) MSN, (B) MSNN-MTX, (C) MSNN-MTX/MIT, and (D) SEM image of MSNN-MTX/MIT. Scale bar is $50 \mathrm{~nm}$.

Abbreviations: TEM, transmission electron microscopy; MTX, methotrexate; MIT, mitoxantrone; MSN, mesoporous silica nanoparticle; MSNN, amino group-modified MSN; SEM, scanning electron microscopy. 
the surface holes was $\sim 1.5 \mathrm{~nm}$ (Figure $8 \mathrm{C}$ ). It is likely that the covalent modification of MTX resulted in a slight increase in the size of the nanoparticle and that the load of MIT resulted in an obvious decrease in the diameter of their surface holes. It is generally accepted that nanoparticles of 77-116 nm in diameter are suitable for cell uptake and that holes of 1.3-2.5 in diameter benefit drug loading and release. ${ }^{31,32}$ The structure of MSNN-MTX/MIT in solid state was assessed by scanning electron microscopy (HITACHI S-4800, Tokyo, Japan), and these images are also provided (Figure 8D). Scanning electron microscopy images indicate that, in the solid state, MSNN-MTX/MIT exists as well-distributed nanoparticles of $\sim 100 \mathrm{~nm}$ in diameter.

\section{Characterization of MSNN-MTX/MIT}

The chemical structure was explained with IR and DSC (Netzsch, Selb, Germany) spectra. As can be seen in Figure 9, after the conversion of MSN to MSNN, a peak appeared at $3,444 \mathrm{~cm}^{-1}$, suggesting that the surface of MSN was modified by NH groups; after the conversion of MSNN to

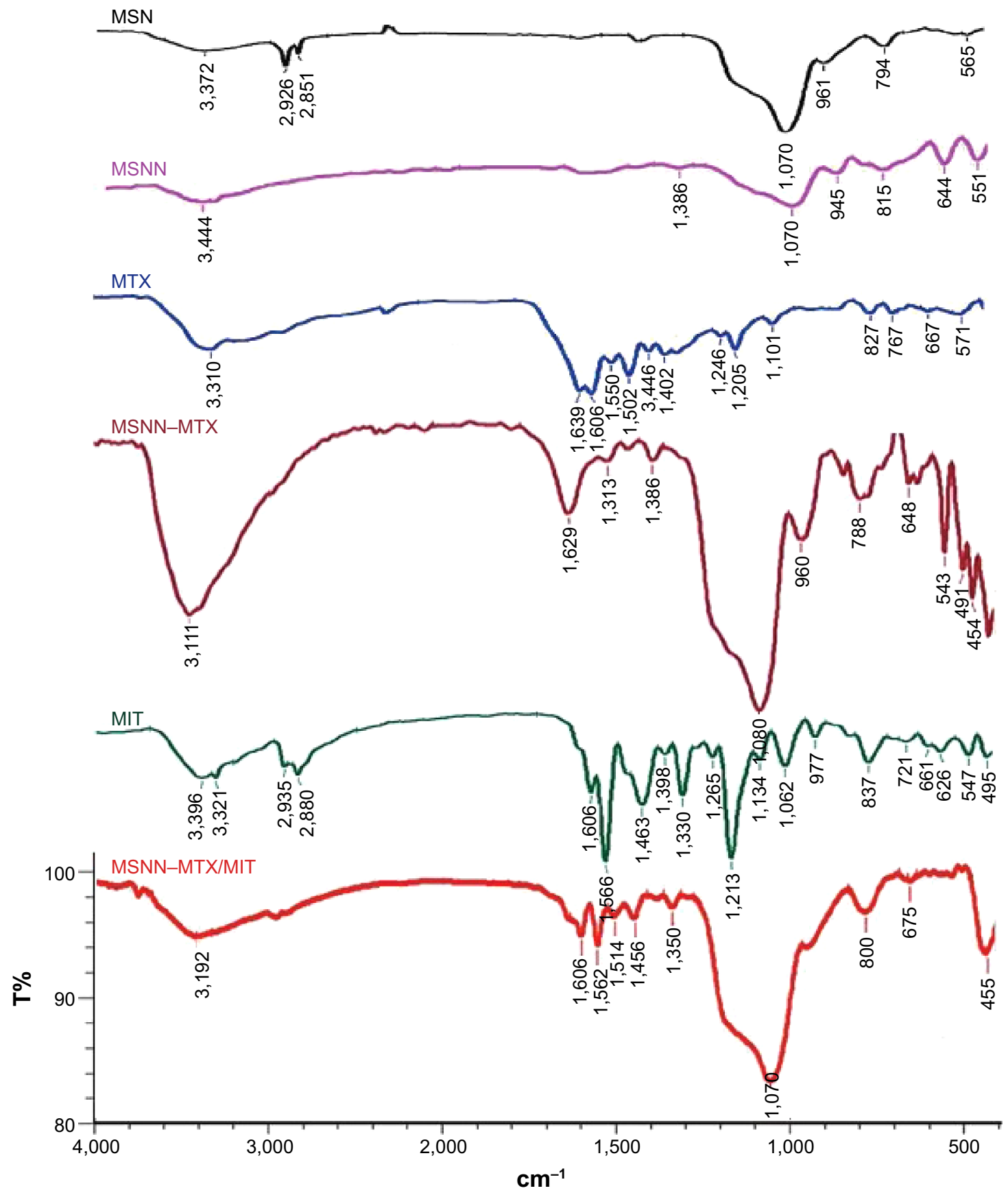

Figure 9 IR spectra of MSN, MSNN, MTX, MIT, MSNN-MTX/MIT, and MSNN-MTX.

Abbreviations: IR, infrared; MTX, methotrexate; MIT, mitoxantrone; MSN, mesoporous silica nanoparticle; MSNN, amino group-modified MSN. 

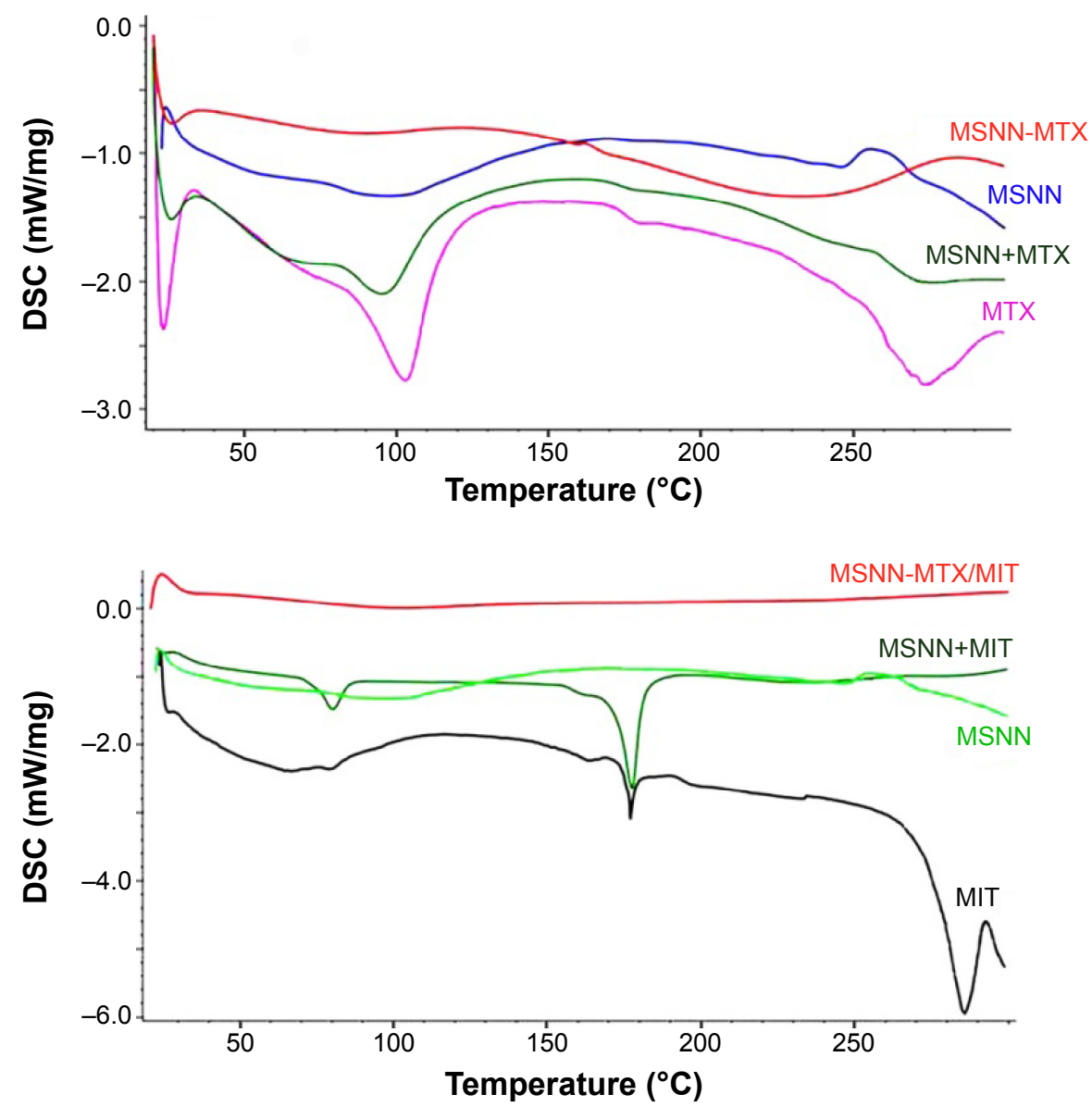

Figure 10 DSC spectra of MSNN, MIT, MTX, MSNN+MTX, MSNN+MIT, MSNN-MTX, and MSNN-MTX/MIT.

Abbreviations: DSC, differential scanning calorimeter; MTX, methotrexate; MIT, mitoxantrone; MSNN, amino group-modified mesoporous silica nanoparticles.

MSNN-MTX, a broad peak appeared at $\sim 2,500$ to $\sim 3,400$ $\mathrm{cm}^{-1}$ and another peak appeared at $1,629 \mathrm{~cm}^{-1}$, suggesting that the $\mathrm{NH}_{2}$ groups on the surface of MSNN were amidated by one carboxyl group of MTX. After the loading of MIT onto the surface holes of MSNN-MTX, the significant peaks of both MIT and MSNN-MTX are involved in the IR spectrum of MSNN-MTX/MIT. Therefore, IR spectra support the reliability of the preparation.

The DSC spectra of MTX and the mixture of MSNN+ MTX are characterized by three peaks at $\sim 25^{\circ} \mathrm{C}, \sim 100^{\circ} \mathrm{C}$, and $\sim 270^{\circ} \mathrm{C}$, whereas the DSC spectrum of MSNN-MTX had no comparable peak (Figure 10), suggesting that covalent modification, but not simple mixing, resulted in MSNN-MTX. The DSC spectra of MIT and the mixture of MSNN+MTX are characterized by two peaks at $\sim 80^{\circ} \mathrm{C}$ and $\sim 180^{\circ} \mathrm{C}$, whereas the DSC spectrum of MSNN-MTX/ MIT had no comparable peak, suggesting that the loading of MIT into the surface holes of MSNN-MTX resulted in the complete integration of MSNN-MTX and MIT. Therefore, DSC spectra also support the reliability of the preparation.

\section{Conclusion}

To prolong the survival period, heighten the cure rate, and lower the systemic toxicity of the usual combination form (MTX/MIT) in treating cancer patients, various combinations of MTX/MIT plus other anticancer drugs have been clinically used. Because all these regimens and the usual combination form (MTX/MIT) have similar limitations, the combination researches remain to be of clinical importance. The in vitro selective release of MTX and MIT in tumor tissue and inside cancer cells and the in vivo prolongation of the survival period, obvious heightening of the cure rate, and significant lowering of the systemic toxicity of the treated S180 mice emphasize that nanostructured MSNN-MTX/ MIT is a promising regimen to alter the usual combination form (MTX/MIT) and various combinations of MTX/MIT plus other anticancer drugs.

\section{Acknowledgments}

This work was supported by the Beijing area major laboratory of peptide and small molecular drugs, by PHR 
(IHLB), by the NSFC (21171120, 81172930, 81270046, and 81373265), Beijing Natural Science Foundation (7132020 and 7132032), Beijing Nova Programme xx2013039, 863 project 2015AA020902, TJSHG201310025008, Project for Science and Technology Development, Beijing Commission of Education (SQKM201210025007), and the Project of Construction of Innovative Teams and Teacher Career Development for Universities and Colleges Under Beijing Municipality. We thank Engineering Research Center of Endogenous Prophylactic of Ministry of Education of China for technical support.

\section{Disclosure}

The authors report no conflicts of interest in this work.

\section{References}

1. Lünenbürger H, Lanvers-Kaminsky C, Lechtape B, Frühwald MC. Systematic analysis of the antiproliferative effects of novel and standard anticancer agents in rhabdoid tumor cell lines. Anticancer Drugs. 2010;21:514-522.

2. Volk EL, Rohde K, Rhee M, et al. Methotrexate cross-resistance in a mitoxantrone-selected multidrug-resistant MCF7 breast cancer cell line is attributable to enhanced energy-dependent drug efflux. Cancer Res. 2000;60(13):3514-3521.

3. Sengupta S, Eavarone D, Capila I, et al. Temporal targeting of tumour cells and neovasculature with a nanoscale delivery system. Nature. 2005;436:568-572.

4. Shin HC, Alani AW, Rao DA, Rockich NC, Kwon GS. Multi-drug loaded polymeric micelles for simultaneous delivery of poorly soluble anticancer drugs. J Control Release. 2009;140(3):294-300.

5. Doughty JC, Kane E, Cooke TG, McArdle CS. Mitoxantrone and methotrexate chemotherapy with and without mitomycin $\mathrm{C}$ in the regional treatment of locally advanced breast cancer. Breast. 2002;11(1):97-99.

6. Kumar A. Effectiveness of mitoxantrone and oral methotrexate combination therapy in relapsing-remitting multiple sclerosis. J Neurol Sci. 2009;285(S1):S155-S339.

7. Kadia TM, Kantarjian HM, Thomas DA, et al. Phase II study of methotrexate, vincristine, pegylated-asparaginase, and dexamethasone (MOpAD) in patients with relapsed/refractory acute lymphoblastic leukemia. Am J Hematol. 2015;90(2):120-124.

8. Ukmar T, Maver U, Planinšek O, Kaučič V, Gaberšček M, Godec A. Understanding controlled drug release from mesoporous silicates: theory and experiment. J Control Release. 2011;155(3):409-417.

9. Rossato LG, Costa VM, Dallegrave E, et al. Cumulative mitoxantroneinduced haematological and hepatic adverse effects in a subchronic in vivo study. Basic Clin Pharmacol Toxicol. 2014;114(3):254-262.

10. Pinto C, Marino A, Manzini VDP, et al. Sequential chemotherapy with cisplatin/gemcitabine (CG) followed by mitoxantrone/methotrexate/ mitomycin (MMM) in patients with malignant pleural mesothelioma. A multicenter Italian Phase II Study (SITMP1). Lung Cancer. 2006; 52(2):199-206.

11. Stein RC, Bower M, Law M, et al. Mitozantrone and methotrexate chemotherapy with and without mitomycin $\mathrm{C}$ in the treatment of advanced breast cancer: a randomised clinical trial. European Journal of Cancer. 1992; 28A(12):1963-1965.

12. Pinto C, Marino A, Guaraldi M, et al. Combination chemotherapy with mitoxantrone, methotrexate, and mitomycin (MMM Regimen) in malignant pleural mesothelioma: a phase II study. Am J Clin Oncol. 2001; 24(2):143-147.

13. Harper-Wynne C, English J, Meyer L, et al. Randomized trial to compare the efficacy and toxicity of cyclophosphamide, methotrexate and 5-fluorouracil (CMF) with methotrexate mitoxantrone (MM) in advanced carcinoma of the breast. Br J Cancer. 1999;81(2):316-322.
14. Kakolyris S, Samonis G, Koukourakis M, et al. First-line treatment with mitoxantrone, methotrexate, vincristine, and carboplatine (MIMOC) plus cyclical hormonotherapy with tamoxifen and megestrol acetate in advanced breast cancer. Am J Clin Oncol. 1999;22(3): 273-277.

15. Lee CK, Gebski VJ, Coates AS, et al. Tradeoffs in quality of life and survival with chemo-therapy for advanced breast cancer: mature results of a randomized trial comparing single-agent mitoxantrone with combination cyclophosphamide, methotrexate, 5-fluorouracil and prednisone. Springerplus. 2013;2:391-400.

16. Di Bona E, Pogliani E, Rossi G, et al. Transplant-finalized salvage of adult acute lymphoblastic leukemia: results of a mitoxantrone and methotrexate-based regimen in 36 patients. Leuk Lymphoma. 2005;46(6): 879-884.

17. Rigacci L, Carrai V, Nassi L, et al. Combined chemotherapy with carmustine, doxorubicin, etoposide, vincristine, and cyclophosphamide plus mitoxantrone, cytarabine and methotrexate with citrovorum factor for the treatment of aggressive non-Hodgkin lymphoma: a long-term follow-up study. Cancer. 2005;103(5):970-977.

18. Pectasides D, Mylonakis A, Antoniou F, et al. Chemotherapy with methotrexate, carbo-platin, mitoxantrone (Novantrone) and vincristine (Oncovin) in transitional-cell urothelial cancer. Oncology. 1998;55(2): 139-144.

19. Raina V, Sharma A, Deo SV, et al. Mitoxantrone, mitomycin-C, methotrexate combination chemotherapy with radiotherapy and/or surgery in stage III (T4B, NO-2, M0) breast cancer. $J$ Assoc Physicians India. 1998;46(11):926-929.

20. van Outryve S, Schrijvers D, van den Brande J, et al. Methotrexateassociated liver toxicity in a patient with breast cancer: case report and literature review. Neth J Med. 2002;60(5):216-222.

21. Conway D, Cohen JA. Combination therapy in multiple sclerosis. Lancet Neurol. 2010;9(3):299-308.

22. Lammers T. Improving the efficacy of combined modality anticancer therapy using HPMA copolymer-based nanomedicine formulations. Adv Drug Deliv Rev. 2010;62(2):203-230.

23. Krishnamachari Y, Salem AK. Innovative strategies for codelivering antigens and CpG oligonucleotides. Adv Drug Deliv Rev. 2009;61(3): 205-217.

24. Pasquier E, Kavallaris M, André N. Metronomic chemotherapy: new rationale for new directions. Nature Reviews Clinical Oncology. 2010;7(8):455-465.

25. Masci G, Losurdo A, Gandini C, et al. Low-dose "metronomic chemotherapy" with oral cyclophosphamide and methotrexate in metastatic breast cancer: a case report of extraordinarily prolonged clinical benefit. Ecancermedicalscience. 2012;6(1):275.

26. Ma TY, Liu L, Yuan ZY. Direct synthesis of ordered mesoporous carbons. Chem Soc Rev. 2013;42(9):3977-4003.

27. Giménez-Bonafé P, Tortosa A, Pérez-Tomás R. Overcoming drug resistance by enhancing apoptosis of tumor cells. Curr Cancer Drug Targets. 2009;9(3):320-340.

28. Kunjachan S, Błauż A, Möckel D. Overcoming cellular multidrug resistance using classical nanomedicine formulations. European Journal of Pharmaceutical Sciences Official Journal of the European Federation for Pharmaceutical Sciences. 2012;45(4):421-428.

29. Yada K, Shibata K, Matsumoto T, Ohta M, Yokoyama S, Kitano S. Protease-activated receptor-2 regulates cell proliferation and enhances cyclooxygenase- 2 mRNA expression in human pancreatic cancer cells. J Surg Oncol. 2005;89(2):79-85.

30. Pimenta DC, Chen VC, Chao J, et al. Alpha1-antichymotrypsin and kallistatin hydrolysis by human cathepsin D. Journal of Protein Chemistry. 2000;19(5):411-418.

31. Chung TH, Wu SH, Yao M, et al. The effect of surface charge on the uptake and biological function of mesoporous silica nano-particles in 3T3-L1 cells and human mesenchymal stem cells. Biomaterials. 2007; 28(19):2959-2966.

32. Huang X, Teng X, Chen D, Tang F, He J. The effect of the shape of mesoporous silica nanoparticles on cellular uptake and cell function. Biomaterials. 2010;31(3):438-448. 


\section{Publish your work in this journal}

Drug Design, Development and Therapy is an international, peerreviewed open-access journal that spans the spectrum of drug design and development through to clinical applications. Clinical outcomes, patient safety, and programs for the development and effective, safe, and sustained use of medicines are the features of the journal, which has also been accepted for indexing on PubMed Central. The manuscript management system is completely online and includes a very quick and fair peer-review system, which is all easy to use. Visit http://www.dovepress.com/testimonials.php to read real quotes from published authors.

Submit your manuscript here: http://www.dovepress.com/drug-design-development-and-therapy-journal 